\title{
Desempeño de biomarcadores de las imágenes con tensor de difusión en regiones tumorales del glioblastoma. Análisis exploratorio de datos mediante gráficos de zombie y pruebas de diagnóstico
}

\author{
Ernesto Roldán-Valadez, ${ }^{*}$ Sofía Anaya-Sánchez,2 Nathaly Rivera-Sotelo' y Sergio Moreno-Jiménez ${ }^{3}$ \\ 'Dirección de Investigación, Hospital General de Mexico "Dr. Eduardo Liceaga", Ciudad de México; 'Escuela de Medicina, Puebla, Universidad de
} las Américas, Puebla, Puebla; ${ }^{3}$ Departamento de Neurocirugía, Instituto Nacional de Neurología y Neurocirugía, Ciudad de México. México

\section{Resumen}

Introducción: Han sido reportados 11 biomarcadores de imágenes con tensor de difusión (DTI) en las regiones tumorales del glioblastoma. Objetivo: Comparar la eficacia de biomarcadores de glioblastoma mediante gráficos de zombie, que permiten la comparación simultánea en función de razones de verosimilitud. Métodos: Cohorte retrospectiva de 29 sujetos con glioblastoma a quienes se efectuó resonancia magnética cerebral de 3 T. Los eigenvalores mayor, intermedio y menor de ITD se utilizaron para calcular 11 biomarcadores en cinco regiones tumorales: sustancia blanca de apariencia normal (NAWM), edema proximal y distal, tumoral viable y necrosis. Las tablas de contingencia con resultados verdaderos y falsos positivos y negativos permitieron calcular gráficos de zombie basados en el factor de Bayes y pruebas diagnósticas previamente no reportadas. Resultados: Los biomarcadores $D M, A F, q, L, C l, C p, A R$ actúan en la zona óptima para el diagnóstico de NAWM. Las regiones de edema proximal y distal, tejido tumoral que se realza con contraste y necrosis no poseen biomarcadores que las identifiquen en un nivel de rendimiento óptimo. Conclusiones: Los biomarcadores DM, AF, q, L, Cl, Cp, $A R$ discriminan el tejido cerebral normal en la zona óptima, pero el rendimiento de otras regiones tumorales se ubica en las zonas de inclusión diagnóstica, exclusión diagnóstica y mediocre.

PALABRAS CLAVE: Neoplasias cerebrales. Imágenes con tensor de difusión. Razón de verosimilitud. Curva ROC. Sensibilidad y especificidad.

\section{Diffusion tensor imaging-derived biomarkers performance in glioblastoma tumor regions: exploratory data analysis using zombie plots and diagnostic tests}

\begin{abstract}
Introduction: Using diffusion tensor imaging (DTI), 11 biomarkers have been reported in different glioblastoma regions. Objective: To compare the efficacy of glioblastoma biomarkers using "zombie plots". Methods: Retrospective cohort of 29 subjects with glioblastoma who underwent 3-Tesla brain magnetic resonance imaging. DTI major, intermediate and minor eigenvalues were used to calculate biomarkers at five tumor regions: normal-appearing white matter (NAWM), proximal and distal edema, tumor tissue and necrosis. Contingency tables with true and false positive and negative results allowed the calculation of zombie plots based on the Bayes factor and previously unreported diagnostic tests. Results: The MD, FA, $q$, $\mathrm{L}, \mathrm{Cl}, \mathrm{Cp}$ and RA biomarkers had a good performance at the optimal zone for NAWM diagnosis. The proximal and distal
\end{abstract}

Correspondencia:

*Ernesto Roldán-Valadez

E-mail: ernest.roldan@usa.net

0016-3813/@ 2021 Academia Nacional de Medicina de México, A.C. Publicado por Permanyer. Este es un artículo open access bajo la licencia CC BY-NC-ND (http://creativecommons.org/licenses/by-nc-nd/4.0/).

Fecha de recepción: 10-09-2021

Fecha de aceptación: 30-09-2021

A.C. Publicado por Permanyer. Este es un artículo open access bajo la licencia
Gac Med Mex. 2022;158:57-65

Disponible en PubMed 
edema, enhancing rim and necrosis regions do not have biomarkers that identify them with an optimal performance level. Conclusions: Zombie plots allow simultaneous comparison of biomarkers based on likelihood ratios. MD, FA, q, L, Cl, Cp, RA discriminated NAWM normal brain tissue at the optimal zone, but performance for other regions was at the mediocre, diagnostic inclusion and diagnostic exclusion zones.

KEYWORDS: Brain neoplasms. Diffusion tensor imaging. Likelihood ratio. ROC curve. Sensitivity and specificity.

\section{Introducción}

Los biomarcadores obtenidos con tensor de difusión, un tipo de imagen de resonancia magnética, han demostrado mayor precisión diagnóstica que la resonancia magnética convencional para diferenciar el glioblastoma. ${ }^{1}$ Se han reportado 11 biomarcadores de glioblastoma en las imágenes con tensor de difusión (ITD): ${ }^{2}$

- Difusividad media (DM).

- Anisotropía fraccionada (AF).

- Difusión isotrópica pura $(p)$.

- Difusión anisotrópica pura (q).

- Magnitud total del tensor de difusión (L).

- Tensor lineal $(\mathrm{Cl})$.

- Tensor plano (Cp).

- Tensor esférico (Cs).

- Anisotropía relativa (AR).

- Difusividad axial (DA).

- Difusividad radial (DR).

En 2016, se dio a conocer el gráfico de zombie como una técnica de análisis exploratorio de datos que permite graficar sensibilidad y especificidad de las pruebas de imagen en función de las razones de verosimilitud (likelihood ratios). ${ }^{3}$

En este estudio se comparó la capacidad diagnóstica de biomarcadores de ITD para la identificación de regiones tumorales del glioblastoma, utilizando gráficos de zombie y pruebas diagnósticas adicionales no publicadas anteriormente. Nuestra hipótesis nula consideró que no habría superioridad de ninguna métrica de ITD; como hipótesis alternativa se consideró que al menos tres biomarcadores tuvieran el rendimiento máximo para su uso en la práctica clínica.

\section{Métodos}

Cohorte retrospectiva de 29 pacientes con diagnóstico patológico de glioblastoma (criterios de la Organización Mundial de la Salud). Los criterios de inclusión consideraron pacientes de uno y otro sexo, mayores a 18 años, evaluados entre enero de 2014 y diciembre de 2018. Los criterios de exclusión incluyeron el tratamiento con corticosteroides o antibióticos, zonas tumorales con calcificación, hemorragia o cirugía cerebral previa. Se excluyeron las exploraciones por resonancia magnética con otras anomalías estructurales. Los comités de ética e investigación institucionales aprobaron el estudio.

\section{Adquisición de imágenes cerebrales}

Se realizó resonancia magnética cerebral en un escáner de $3 \mathrm{~T}$ (Signa HDxt, GE Healthcare, Waukesha, WI, Estados Unidos), con bobina de cráneo de ocho canales de alta resolución (Invivo, Gainesville, FL, Estados Unidos). Una descripción detallada del protocolo en la resonancia magnética ha sido publicada recientemente por nuestro grupo. ${ }^{4}$

\section{Regiones tumorales seleccionadas y mediciones derivadas de ITD}

Conforme la metodología descrita, ${ }^{2}$ se calcularon 11 biomarcadores de ITD en cinco regiones tumorales:

- NAWM (sustancia blanca de apariencia normal).

- Anillo tumoral con realce al contraste.

- Edema proximal.

- Edema distal.

- Necrosis.

La Figura 1 muestra la imagen ponderada en T1-w previa al contraste, la T1-w posterior al contraste y T2 (T2-w) que identifican las regiones tumorales seleccionadas.

\section{Análisis estadístico}

En la Figura 2 se describe el diseño de los gráficos de zombie. Las evaluaciones de pruebas de diagnóstico y el software utilizado se detallan en un archivo complementario de acceso en línea (https://doi. org/10.24875/GMM.21000583) 

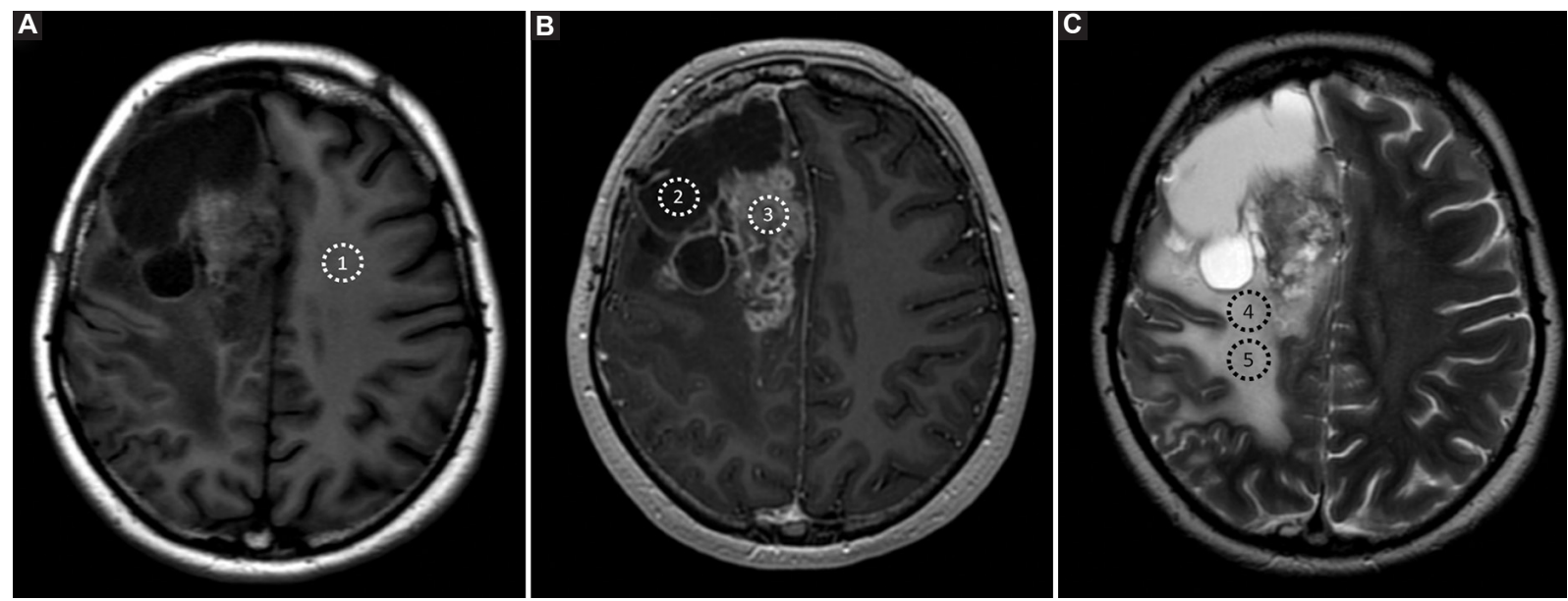

Figura 1. Secuencias convencionales de resonancia magnética para localizar regiones tumorales. A: imagen ponderada en T1-w previa al contraste, en la que se localizó la sustancia blanca de apariencia normal (NAWM). B: imagen en T1-w poscontraste que muestra el realce del anillo tumoral y la zona central de necrosis. C: imagen ponderada en T2 (T2-w) en la que se identificó edema proximal y edema distal.

A

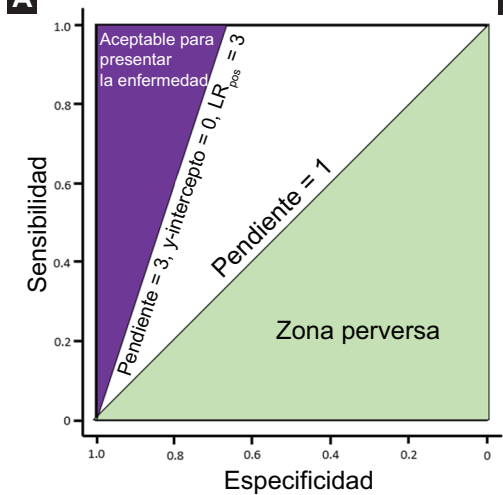

B

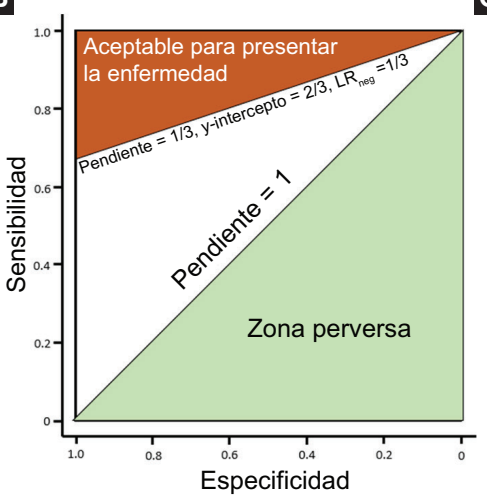

C

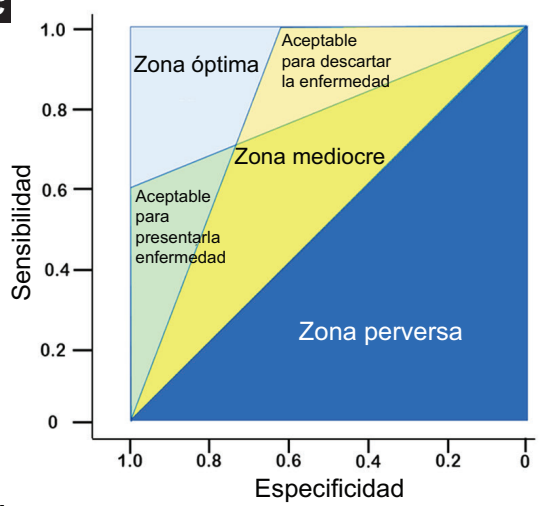

Figura 2. Gráficas de ROC con las tres líneas de razón de verosimilitud constante que componen las zonas del gráfico de zombie. A: línea de identidad con una pendiente de 1; el área debajo de esta línea se ha denominado "zona perversa"; el área por encima de la línea, donde la pendiente es 3, proporciona un rendimiento aceptable de presentar la enfermedad. B: la zona perversa está debajo de la línea donde la pendiente es 1; el área por encima de la línea, donde la pendiente es de 1/3 proporciona un rendimiento aceptable para descartar enfermedades. C: el gráfico de ROC muestra las cinco zonas del gráfico de zombie (zonas de mayor eficacia de imagen deficiente). El área en forma de bumerán, integrada por zonas azul, verde y naranja, establece la zona de eficacia aceptable.

\section{Resultados}

\section{Desempeño de los biomarcadores de ITD determinado con gráficos de zombie}

En la región NAWM, siete biomarcadores se ubicaron en la "zona óptima" para el diagnóstico (DM, AF, $q, \mathrm{~L}, \mathrm{Cl}, \mathrm{Cp}, \mathrm{AR})$; tres biomarcadores estaban en la "zona de exclusión diagnóstica" (Cs, DR, DA) y solo uno estuvo en la "zona de inclusión diagnóstica" (p). Ninguna otra región tumoral tuvo algún biomarcador en la zona óptima para el diagnóstico. La región tumoral tuvo seis biomarcadores en la zona de exclusión diagnóstica (DM, p, q, L, Cp, DR, DA); dos biomarcadores en la "zona mediocre" (AF, $\mathrm{Cl}$ ) y dos en la "zona perversa" (Cs, AR).

La Tabla 1 muestra el desempeño de los biomarcadores de ITD en función de la localización de las razones de verosimilitud agrupadas por regiones en los gráficos de zombie.

Las Figuras 3 y 4 representan los gráficos de zombie correspondientes a cada biomarcador ITD, los cuales tiene un punto de operación único y $95 \%$ de intervalo de confianza para la sensibilidad y especificidad, que se representa con áreas elípticas de colores. 
Gaceta Médica de México. 2022;158

Tabla 1. Desempeño de los biomarcadores de ITD según la localización de sus razones de verosimilitud en las regiones del gráfico de zombie

\begin{tabular}{|c|c|c|c|c|c|}
\hline Región del gráfico & NAWM & Edema proximal & Edema distal & Tumor viable & Necrosis \\
\hline Zona óptima para el diagnóstico & $\begin{array}{l}\mathrm{DM}, \mathrm{AF}, \mathrm{q}, \mathrm{L}, \\
\mathrm{Cl}, \mathrm{Cp}, \mathrm{AR}\end{array}$ & & & & \\
\hline Zona incluyente para el diagnóstico & Cs, DR, DA & $\begin{array}{l}\text { DM, AF, p, q, L, } \\
\text { Cp, Cs, AR, DA }\end{array}$ & $\begin{array}{l}\mathrm{DM}, \mathrm{AF}, \mathrm{p}, \mathrm{q}, \mathrm{L}, \mathrm{Cl} \\
\mathrm{Cp}, \mathrm{Cs}, \mathrm{AR}, \mathrm{DR}\end{array}$ & $\begin{array}{l}\mathrm{DM}, \mathrm{p}, \mathrm{q}, \mathrm{L}, \mathrm{Cp} \\
\mathrm{DR}, \mathrm{DA}\end{array}$ & \\
\hline Zona excluyente para el diagnóstico & $p$ & & & & $A F, q, C p, D A$ \\
\hline Zona mediocre & & $\mathrm{Cl}, \mathrm{DR}$ & DA & $\mathrm{AF}, \mathrm{Cl}$ & DM, p, Cs, AR, DR \\
\hline Zona perversa & & & & Cs, AR & $\mathrm{L}, \mathrm{Cl}$ \\
\hline
\end{tabular}
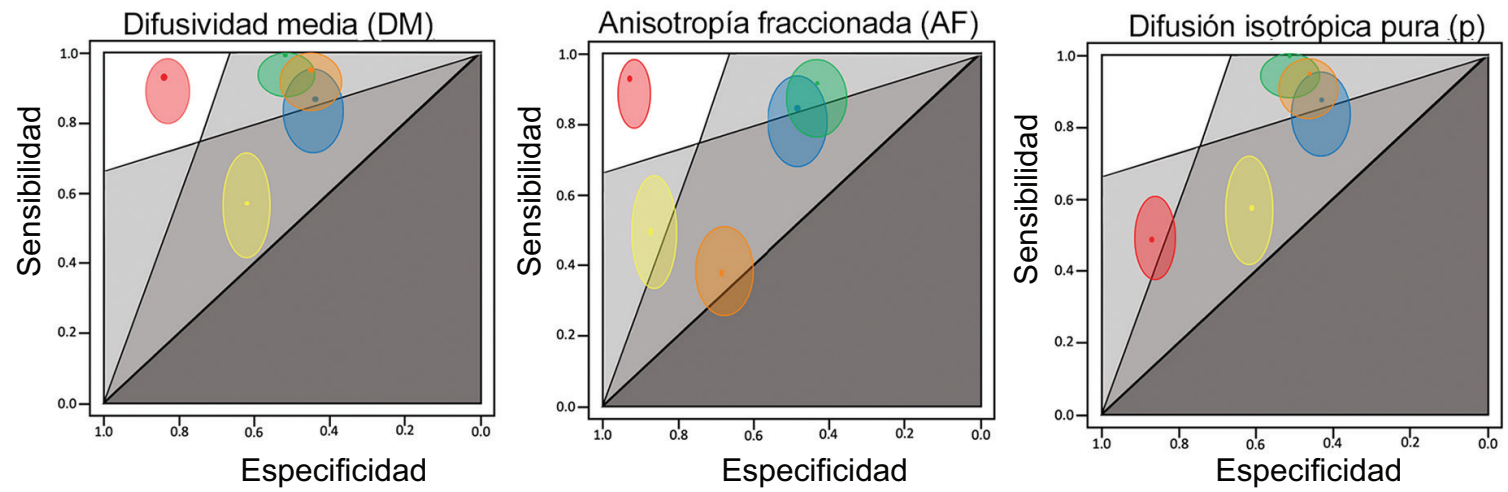

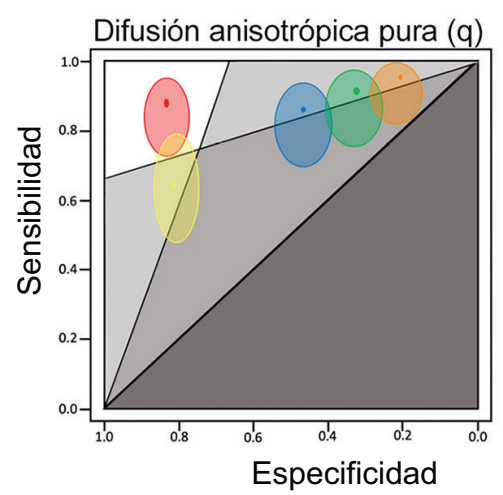

Región normal

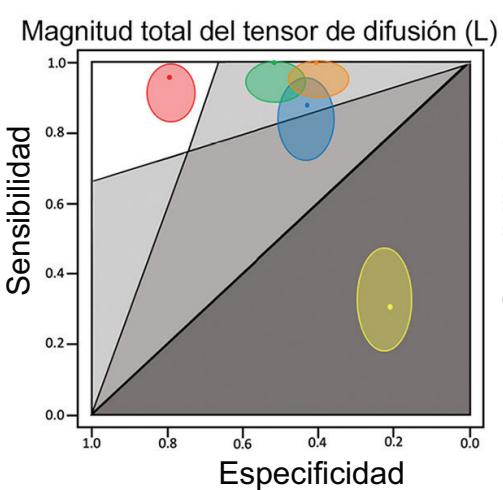

Edema proximal

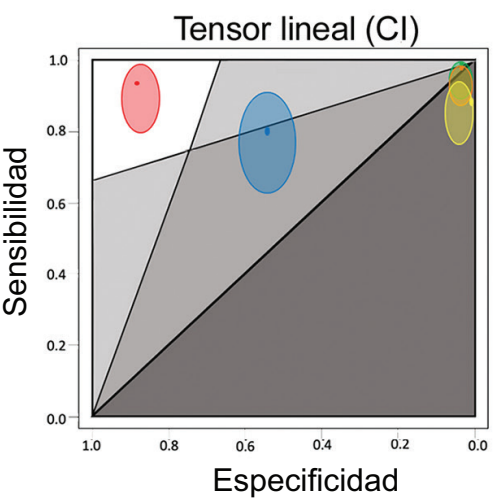

Edema distal

Región de necrosis

Región tumoral

Figura 3. Gráfico de zombie que muestra el punto de operación y su intervalo de confianza de 95 para difusividad media (DM), anisotropía fraccionada $(A F)$, difusión isotrópica pura (p), difusión anisotrópica pura (q), magnitud total del tensor de difusión ( $L$ ) y tensor lineal $(C l)$. Las áreas en forma de elipse de colores corresponden a NAWM (rojo), edema proximal (azul), edema distal (verde), necrosis (amarillo) y borde realzado o región tumoral (naranja).

\section{Rendimiento basado en el área bajo la curva ROC (AUROC)}

Solo la región NAWM representó biomarcadores con excelente desempeño diagnóstico AUROC (AF, DR,
$\mathrm{AR}, \mathrm{q}, \mathrm{Cs}, \mathrm{Cl}$ ) y bueno (p, DM, L, Cp). Los biomarcadores con mejor desempeño para detectar la región tumoral solo ofrecieron AUROC deficiente (DA, DR, $p$, $\mathrm{DM}, \mathrm{L}, \mathrm{Cp}$ ); incluso, el resto fracasó en el diagnóstico (AF, AR, q, Cs, Cl). La mayoría de los biomarcadores 

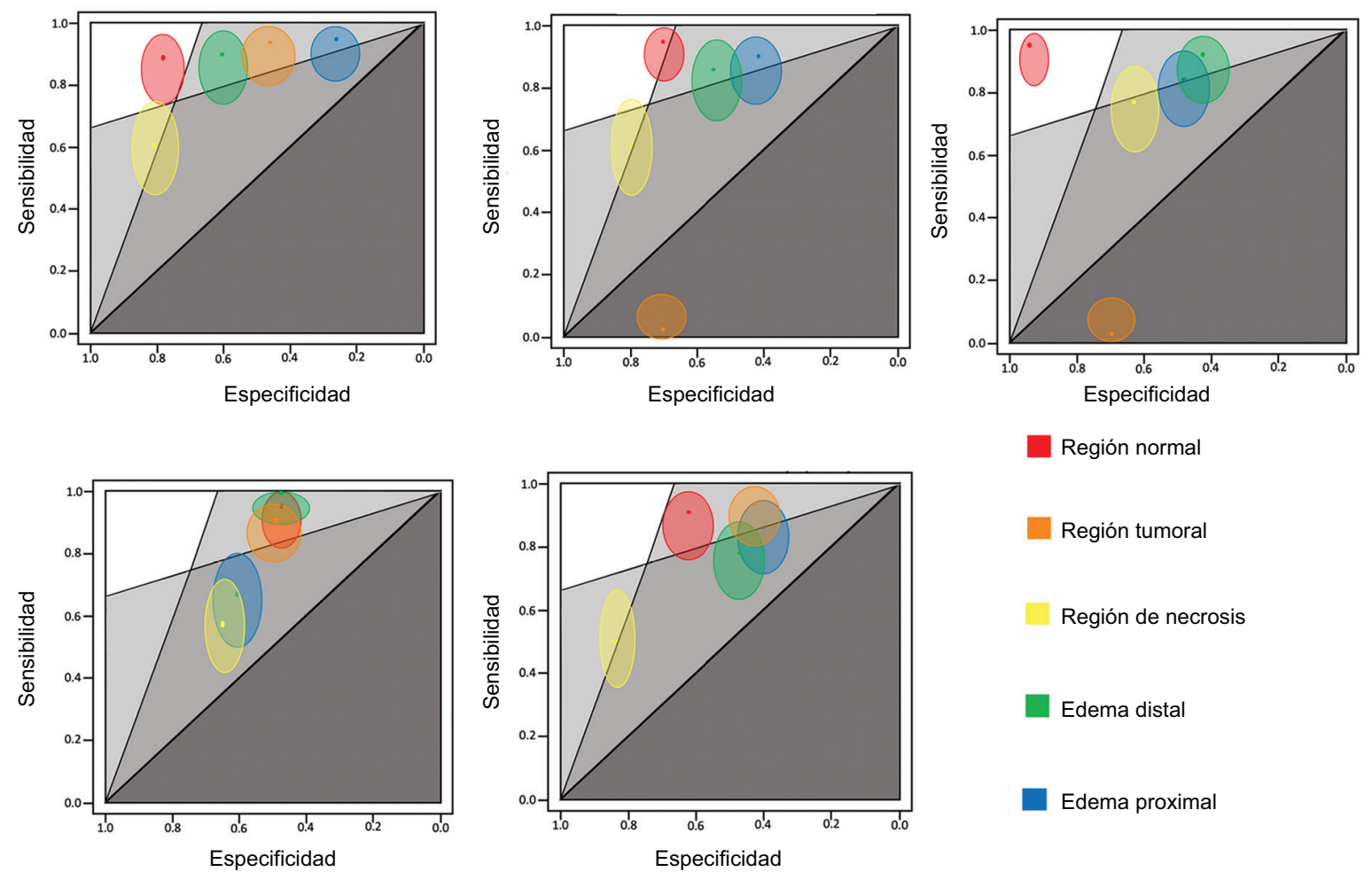

Región normal

Región tumoral

Región de necrosis

Edema distal

Edema proximal

Figura 4. Gráfico de zombie combinado que muestra el punto de operación y su intervalo de confianza de 95 \% para tensor plano (Cp), tensor esférico (Cs), anisotropía relativa (AR), difusividad radial (DR) y difusividad axial (DA). Las áreas en forma de elipse de colores corresponden a NAWM (rojo), edema proximal (azul), edema distal (verde), necrosis (amarillo) y borde realzado o región tumoral (naranja).

capaces de detectar edema proximal y distal mostraron un mal desempeño.

La Tabla 2 muestra el rendimiento de los biomarcadores ITD seleccionados en función del AUROC (excelente, buena, regular, deficiente y fallida) y los agrupa por regiones tumorales.

\section{Rendimientos adicionales de las pruebas de diagnóstico}

\section{Precisión diagnóstica global (eficacia)}

La proporción de elementos correctamente clasificados tuvo valores más altos (> $90 \%$ ) en la detección de NAWM (AF, Cs, AR y DR). DM fue el único biomarcador capaz de alcanzar un rango de 80 a $90 \%$ en la detección de la región tumoral. Las regiones de edema proximal y edema distal solo presentaron biomarcadores con desempeño deficiente o nulo (por debajo de $70 \%$ ). En la Tabla 3 se detalla el rendimiento diagnóstico basado en la precisión diagnóstica general.

\section{RAZÓN DE MOMIOS DE DIAGNÓSTICO}

Solo cuatro biomarcadores presentaron los valores más altos para detectar NAWM (Cs, AR, DR y AF). En comparación con los valores de la región NAWM, los valores de las regiones tumoral, de edema proximal y edema distal fueron muy bajos. Los valores de los biomarcadores para detectar la región de necrosis fueron ligeramente más altos, pero aún bajos en comparación con los de NAWM. En la Tabla 4 se muestra el rendimiento diagnóstico de los biomarcadores de ITD utilizando la definición de razón de momios para el diagnóstico.

\section{Índice de Youden}

Los mayores valores positivos correspondieron a NAWM (Cs, AR, DR, AF y Cl). Por el contrario, los valores más bajos se encontraron en las regiones tumoral, de edema distal y necrosis medidos con el biomarcador Cl. La Tabla 5 muestra el rendimiento diagnóstico de los biomarcadores de ITD utilizando la fórmula del índice de Youden. 
Tabla 2. Área bajo la curva ROC de los 11 biomarcadores de ITD agrupados por regiones tumorales

\begin{tabular}{|c|c|c|c|c|c|}
\hline $\begin{array}{l}\text { Relación entre la curva ROC y } \\
\text { el desempeño diagnóstico }\end{array}$ & NAWM & Edema proximal & Edema distal & Tumor viable & Necrosis \\
\hline $0.90-1$, excelente & $\begin{array}{l}A F, D R, A R, q, \\
C s, C l\end{array}$ & & & & \\
\hline 0.80-0.90, bueno & $p, D M, L, C p$ & & & & \\
\hline $0.70-0.80$, regular & DA & & $\mathrm{Cp}$ & & $\begin{array}{l}\text { DA, AF, DR, AR, p, } \\
\text { DM, L, Cs, Cp, Cl }\end{array}$ \\
\hline $0.60-0.70$, pobre & & $\begin{array}{l}A F, D R, A R, p, D M, ~ L, \\
C s, C l\end{array}$ & $\begin{array}{l}\mathrm{DA}, \mathrm{AF}, \mathrm{DR}, \mathrm{AR}, \mathrm{p} \\
\mathrm{DM}, \mathrm{L}, \mathrm{Cs}\end{array}$ & $\begin{array}{l}\mathrm{DA}, \mathrm{DR}, \mathrm{p}, \mathrm{DM}, \\
\mathrm{L}, \mathrm{Cp}\end{array}$ & $q$ \\
\hline 0.50-0.60, fallido & & $\mathrm{DA}, \mathrm{q}, \mathrm{Cp}$ & $\mathrm{Q}, \mathrm{Cl}$ & $\mathrm{AF}, \mathrm{AR}, \mathrm{q}, \mathrm{Cs}, \mathrm{Cl}$ & \\
\hline
\end{tabular}

ITD: imágenes con tensor de difusión; NAWM: sustancia blanca de apariencia normal.

Tabla 3. Desempeño diagnóstico de los biomarcadores de ITD utilizando la precisión diagnóstica general

\begin{tabular}{|l|l|l|l|l|l|}
\hline Biomarcador de ITD & NAWM & Edema proximal & Edema distal & Tumor viable & Necrosis \\
\hline Difusividad media & 0.8607 & 0.5224 & 0.6020 & 0.8607 & 0.8657 \\
\hline Anisotropía fraccionada & 0.9303 & 0.5500 & 0.5155 & 0.5522 & 0.8000 \\
\hline Difusión isotrópica pura & 0.8756 & 0.5174 & 0.6020 & 0.5672 & 0.8657 \\
\hline Difusión anisotrópica pura & 0.8458 & 0.5423 & 0.4378 & 0.3831 & 0.7811 \\
\hline Magnitud total del tensor de difusión & 0.8308 & 0.5174 & 0.6020 & 0.5224 & 0.2338 \\
\hline Tensor lineal & 0.8955 & 0.5920 & 0.1990 & 0.2289 & 0.1891 \\
\hline Tensor plano & 0.8060 & 0.3980 & 0.6617 & 0.5672 & 0.7711 \\
\hline Tensor esférico & 0.9602 & 0.5124 & 0.6020 & 0.5672 & 0.7662 \\
\hline Anisotropía relativa & 0.9403 & 0.5522 & 0.5174 & 0.5672 & 0.6567 \\
\hline Difusividad axial & 0.6866 & 0.4925 & 0.5572 & 0.5274 & 0.8806 \\
\hline Difusividad radial & 0.9154 & 0.6219 & 0.5721 & 0.5821 & 0.8706 \\
\hline ITD: imágenes con tensor de difusión; NAWM: sustancia blanca de apariencia normal. & & &
\end{tabular}

\section{Discusión}

El gráfico de zombie permitió comparar simultáneamente la eficacia de la imagen basada en las razones de verosimilitud de 11 biomarcadores de imagen. En este estudio confirmamos nuestra hipótesis alternativa: al menos tres biomarcadores tuvieron un desempeño en la zona óptima de diagnóstico.

La relevancia clínica de nuestro estudio tiene varios componentes:

1. Identificamos siete biomarcadores (DM, AF, q, L, $\mathrm{Cl}, \mathrm{Cp}, \mathrm{AR})$ que funcionan en la zona óptima, pero solo para el diagnóstico de NAWM.

2. Las regiones tumorales correspondientes a edema proximal y distal, la zona de tumor que se realza con el contraste y las regiones de necrosis no tuvieron biomarcadores capaces de identificarlas en un nivel óptimo de rendimiento.

3. La identificación de las regiones tumoral y de edema proximal y distal es posible por al menos siete biomarcadores en cada una, pero solo para descartar el diagnóstico (Tabla 1).

4. Solo cuatro biomarcadores (AF, q, Cp, DA) lograron identificar la región de necrosis.

5. Cuando los biomarcadores ITD se clasifican en función de sus áreas bajo la curva ROC, el NAWM vuelve a ser la única región con biomarcadores que se desempeñan en la zona óptima. Sin embargo, la selección de biomarcadores es ligeramente diferente a la selección del gráfico de zombie. 
Tabla 4. Desempeño diagnóstico de los biomarcadores de ITD utilizando la razón de momios de diagnóstico

\begin{tabular}{|c|c|c|c|c|c|}
\hline Biomarcador de ITD & NAWM & Edema proximal & Edema distal & Tumor viable & Necrosis \\
\hline Difusividad media & 69.68 & 5.31 & - & 16.14 & 22.22 \\
\hline Anisotropía fraccionada & 174.91 & 4.95 & 8.06 & 0.11 & 7.30 \\
\hline Difusión isotrópica pura & 62.43 & 5.17 & - & 17.41 & 22.22 \\
\hline Difusión anisotrópica pura & 37.85 & 4.86 & 5.50 & 6.07 & 8.07 \\
\hline Magnitud total del tensor de difusión & 79.38 & 5.17 & - & - & 0.12 \\
\hline Tensor lineal & 101.83 & 4.61 & - & - & - \\
\hline Tensor plano & 27.21 & 6.68 & 12.63 & 11.61 & 6.59 \\
\hline Tensor esférico & 510.00 & 6.33 & 7.44 & 0.06 & 6.71 \\
\hline Anisotropía relativa & 298.00 & 5.11 & 8.31 & 0.06 & 5.42 \\
\hline Difusividad axial & 16.10 & 4.56 & 30.63 & 9.47 & 52.00 \\
\hline Difusividad radial & 192.00 & 3.14 & - & 9.38 & 25.17 \\
\hline
\end{tabular}

Tabla 5. Desempeño diagnóstico de los biomarcadores de ITD utilizando el índice de Youden

\begin{tabular}{|l|l|l|l|l|l|}
\hline Biomarcador de ITD & NAWM & Edema proximal & Edema distal & Tumor viable & Necrosis \\
\hline Difusividad media & 0.7713 & 0.3101 & 0.5152 & 0.3989 & 0.5148 \\
\hline Anisotropía fraccionada & 0.8594 & 0.3236 & 0.3446 & 0.2668 & 0.3864 \\
\hline Difusión isotrópica pura & 0.7727 & 0.3039 & 0.5152 & 0.4178 & 0.5148 \\
\hline Difusión anisotrópica pura & 0.7174 & 0.3153 & 0.2500 & 0.1851 & 0.4605 \\
\hline Magnitud total del tensor de difusión & 0.7511 & 0.3039 & 0.5152 & 0.3962 & 0.4766 \\
\hline Tensor lineal & 0.8154 & 0.3381 & 0.0242 & 0.0252 & 0.0952 \\
\hline Tensor plano & 0.6671 & 0.2142 & 0.5010 & 0.4003 & 0.4128 \\
\hline Tensor esférico & 0.9146 & 0.3172 & 0.4066 & 0.2655 & 0.4241 \\
\hline Anisotropía relativa & 0.8895 & 0.3276 & 0.3470 & 0.2655 & 0.3908 \\
\hline Difusividad axial & 0.5337 & 0.2730 & 0.4389 & 0.3500 & 0.4811 \\
\hline Difusividad radial & 0.8580 & 0.2778 & 0.4788 & 0.4016 & 0.5211 \\
\hline ITD. imágenes con tensor de difusión: NAwM: sustancia blanca de apariencianormal. & & & &
\end{tabular}

Una selección basada en las razones de verosimilitud tiene propiedades poderosas desde la perspectiva clínica. La razón de verosimilitud es la relación entre la probabilidad de un resultado esperado en una prueba en sujetos con la enfermedad y la probabilidad en sujetos sin la enfermedad. ${ }^{5}$

Las métricas de ITD indican la direccionalidad de la difusión del agua, que refleja la cantidad de mielinización en los haces axonales o la integridad de los tractos de fibras. ${ }^{6}$ Además, proporcionan información ultraestructural sobre la densidad celular y las propiedades de la matriz extracelular. ${ }^{7}$

Nuestro estudio previo sobre el desempeño de biomarcadores ITD no presenta una evaluación diagnóstica completa de todas las pruebas diagnósticas conocidas. ${ }^{8}$ Con los gráficos de zombie fue posible la representación visual del desempeño de los biomarcadores en cada región tumoral.

Uno de los principales desafíos en el seguimiento del glioblastoma es identificar cuándo aparecerá una 
nueva área de realce del tumor en la región periférica de este; en $40 \%$ de los casos, la infiltración tumoral puede ocurrir en cerebros que parecen normales en imágenes ponderadas en T2. ${ }^{9}$

Debido a que existe una creciente preocupación por la sobreutilización de las pruebas diagnósticas y los marcadores, creemos que el uso de los métodos presentados en este artículo ayudarán a los médicos clínicos a disminuir las posibles consecuencias negativas de las pruebas excesivas y los costos asociados, que cada vez son mayores.

\section{Limitaciones del estudio}

Hay particularidades de algunas pruebas diagnósticas que deben mencionarse. El índice de Youden no es sensible a las diferencias en la sensibilidad y especificidad de la prueba, lo que constituye su principal desventaja. Además, no se ve afectado por la prevalencia de la enfermedad, pero sí por el espectro de la enfermedad; lo mismo pasa con la sensibilidad, la especificidad, las razones de verosimilitud y la razón de momios de diagnóstico. ${ }^{10}$

Por otro lado, se utilizó una serie no consecutiva de pacientes, lo cual se ha asociado a sobreestimación de $50 \%$ en la razón de momios de diagnóstico, en comparación a cuando se emplea una serie consecutiva de pacientes. ${ }^{10}$

\section{Direcciones futuras}

El mayor número de hallazgos basados en datos de imágenes amplía en gran medida la visión del médico para evaluar la patogénesis del glioma y las estrategias terapéuticas: ${ }^{11}$ otras técnicas avanzadas de imágenes, incluidas las imágenes ponderadas por perfusión y la espectroscopia de resonancia magnética, podrían complementar la evaluación de la infiltración tumoral' ${ }^{12}$ y el diseño de modelos de predicción de la progresión tumoral o de la presencia de células tumorales en el edema peritumoral, lo que permitiría disminuir la variabilidad pronóstica de paciente a paciente. ${ }^{4} \mathrm{El}$ análisis multivariado de métricas de ITD que controlen el efecto de factores de confusión (sexo, edad, ubicación regional del tumor, patrones de infiltración mediante ITD) podría revelar interacciones desconocidas de los biomarcadores a nivel ultraestructural en los glioblastomas, para revelar aplicaciones clínicas adicionales.

Los protocolos avanzados de resonancia magnética, incluido las ITD, se han incorporado en algunas instituciones a los protocolos de diagnóstico de gliomas mediante imágenes..$^{13}$ Creemos que la inteligencia artificial, el aprendizaje automático, el diagnóstico asistido por computadora y la radiómica cambiarán la forma como trabajan los radiólogos y otros especialistas en imágenes y, probablemente, muy pronto, cambiarán la perspectiva que las diferentes especialidades tienen sobre su trabajo. ${ }^{14}$ Sin duda, la inteligencia artificial podría "reducir el retraso" de los estudios de imagen, simplificar la interpretación y la presentación de informes, hacer el análisis de imágenes más objetivo y reproducible y conducir a la radiología hacia los conceptos de medicina de precisión y evaluación multidisciplinaria del paciente. ${ }^{15}$

\section{Conclusiones}

Nuestros hallazgos en este estudio nos permitieron identificar siete biomarcadores que funcionan a nivel óptimo para detectar sustancia blanca de aspecto normal. Al conocer los parámetros normales, podemos identificar preoperatoriamente los sitios de infiltración tumoral significativa que parecen normales en la resonancia magnética convencional y en el seguimiento de los pacientes con glioblastoma. Las técnicas de aprendizaje automático e inteligencia artificial promoverán la automatización de este método en el futuro próximo, disminuirán los tiempos de posprocesamiento y los errores de interpretación, así como el empirismo subyacente en la adopción de ITD.

\section{Financiamiento}

La presente investigación no recibió ninguna beca específica de agencias de los sectores público, comercial o sin ánimo de lucro.

\section{Conflicto de intereses}

Los autores declaran no tener conflicto de intereses.

\section{Responsabilidades éticas}

Protección de personas y animales. Los autores declaran que para esta investigación no realizaron experimentos en seres humanos ni en animales.

Confidencialidad de los datos. Los autores declaran que en este artículo no aparecen datos de pacientes.

Derecho a la privacidad y consentimiento informado. Los autores declaran que en este artículo no aparecen datos de pacientes. 


\section{Bibliografía}

1. Roldán-Valadez E, Ríos C, Cortez-Conradis D, Favila R Moreno-Jiménez S. Global diffusion tensor imaging derived metrics differentiate glioblastoma multiforme vs. normal brains by using discriminant analysis: introduction of a novel whole-brain approach. Radio Oncol. 2014:48:127-136.

2. Cortez-Conradis D, Favila R, Isaac-Olive K, Martínez-López M, Ríos C, Roldán-Valadez E. Diagnostic performance of regional DTI-derived tensor metrics in glioblastoma multiforme: simultaneous evaluation of $p, q$ $\mathrm{L}, \mathrm{Cl}, \mathrm{Cp}, \mathrm{Cs}, \mathrm{AR}, \mathrm{DR}, \mathrm{DA}$, mean diffusivity and fractional anisotropy. Eur Radiol. 2013;23:1112-1121.

3. Richardson ML. The zombie plot: a simple graphic method for visualizing the efficacy of a diagnostic test. AJR Am J Roentgenol. 2016;207:W43-W52.

4. Flores-Álvarez E, Ríos-Piedra EA, Cruz-Priego GA, Durand-Muñoz C Moreno-Jiménez S, Roldán-Valadez E. Correlations between DTI-derived metrics and MRS metabolites in tumour regions of glioblastoma: a pilot study. Radiol Oncol. 2020;54:394-408.

5. Deeks JJ, Altman DG. Diagnostic tests 4: likelihood ratios. BMJ 2004;329:168-169.

6. Waldman AD, Jackson A, Price SJ, Clark CA, Booth TC, Auer DP, et al. Quantitative imaging biomarkers in neuro-oncology. Nat Rev Clin Oncol. 2009;6:445-454.

7. Sadeghi N, Camby I, Goldman S, Gabius HJ, Baleriaux D, Salmon I, et al. Effect of hydrophilic components of the extracellular matrix on quantifiable diffusion-weighted imaging of human gliomas: preliminary results of correlating apparent diffusion coefficient values and hyaluronan expression level. AJR Am J Roentgenol. 2003;181:235-241.
8. Toh $\mathrm{CH}$, Wei KC, Ng SH, Wan YL, Lin CP, Castillo M. Differentiation of brain abscesses from necrotic glioblastomas and cystic metastatic brain tumors with diffusion tensor imaging. AJNR Am J Neuroradiol. 2011;32:1646-1651.

9. Price SJ, Jena R, Burnet NG, Hutchinson PJ, Dean AF, Pena A, et al. Improved delineation of glioma margins and regions of infiltration with the use of diffusion tensor imaging: an image-guided biopsy study. AJNR Am J Neuroradiol. 2006;27:1969-1974.

10. Rutjes AW, Reitsma JB, Di Nisio M, Smidt N, van Rijn JC, Bossuyt PM. Evidence of bias and variation in diagnostic accuracy studies. CMAJ. 2006;174:469-476.

11. Cao H, Erson-Omay EZ, Gunel M, Moliterno J, Fulbright RK. A quantitative assessment of pre-operative MRI reports in glioma patients: report metrics and IDH prediction ability. Front Oncol. 2020;10:600327.

12. Bette S, Huber T, Gempt J, Boeckh-Behrens T, Wiestler B, Kehl V, et al. Local fractional anisotropy is reduced in areas with tumor recurrence in glioblastoma. Radiology. 2017;283:499-507.

13. Johnson DR, Guerin JB, Giannini C, Morris JM, Eckel LJ, Kaufmann TJ. 2016 updates to the WHO brain tumor classification system: what the radiologist needs to know. Radiographics. 2017;37:2164-2180.

14. Santos MK, Ferreira-Júnior JR, Wada DT, Tenorio APM, Barbosa MHN, Marques PMA. Artificial intelligence, machine learning, computer-aided diagnosis, and radiomics: advances in imaging towards to precision medicine. Radiol Bras. 2019;52:387-396.

15. Moreno-Jiménez S, Gerson-Cwilich R, Poitevin-Chacon A, Carrillo-Ruiz JD, Roldán-Valadez E, Shveid-Gerson D, et al. Medicina de precisión en la imagenología médica, radioncología, oncología médica y neurocirugía. Gac Med Mex. 2021 (en revisión). 